\title{
Evaluation of Measurement Uncertainty of Radiated EMC Tests in Arbitrary Field Generators using Surface Currents on DUT
}

\author{
T. Schrader ${ }^{1}$, K. Münter ${ }^{1}$, M. Spitzer ${ }^{1}$, N. Eulig ${ }^{2}$, and A. Enders ${ }^{2}$ \\ ${ }^{1}$ Physikalisch-Technische Bundesanstalt, AG 2.21, Bundesallee 100, 38116 Braunschweig, Germany \\ ${ }^{2}$ Institut für Elektromagnetische Verträglichkeit, Technische Universität Braunschweig, Schleinitzstraße 23, 38106 \\ Braunschweig, Germany
}

\begin{abstract}
We present a method for the evaluation of measurement uncertainty in radiated EMC tests. It is based on the measurement of surface current densities on a sphere, the results are compared to the surface current distribution on a sphere in a free-space environment obtained by numerical calculations. The free-space is considered to be a reference field generator. By this method we avoid burdening any systematic deviations of one particular (historic) method to any of the others. We evaluate the ratio $\boldsymbol{J} / \boldsymbol{E}$ of the measured surface current density $\boldsymbol{J}$ and the measured empty electrical field strength $\boldsymbol{E}$ for both the reverberation chamber and the semi-anechoic chamber.
\end{abstract}

\section{Introduction}

The test of Electromagnetic Compatibility (EMC) of electrical and electronic devices can be divided into two different parts - the emissions measurement and the susceptibility test. In this paper emphasis is placed on the susceptibility test which can further be subdivided into the line-conducted disturbance test and the field-coupled test. One part of the fieldcoupled test is, speaking generally, an analysis of the reaction of the device under test (DUT) on impinging electromagnetic fields from external sources. Over the last decades many test setups, field generators (FG), and concomitant standards have been developed and are now used throughout the EMC test laboratories worldwide (IEC EN 61000-4-3, -20, and -21) in order to determine the DUT's reaction.

Testing is not done for an end in itself. The standards IEC EN 61000-6-1 (Immunity Residential Area), 61000-6-2 (Immunity Industrial Area), 61000-6-3 (Emission Residential Area), 61000-6-4 (Emission Industrial Area) have set limits for both, emission and disturbance levels. In addition, product committees may have set other limits for their devices. These limits must be met during measurement and

Correspondence to: T. Schrader

(thorsten.schrader@ptb.de) testing using different FGs. Regarding this, one can ask for the comparability of test results obtained in different field generators. All standards claim to provide a technical solution to the given problem of EMC testing, but none of the standards show how to handle measurement uncertainty with respect to the given limits or even to derive a test or measurement uncertainty budget. At the moment there is no confidence about the quality, the informational value and the meaning or evidence of any test result. For susceptibility testing also a representative physical quantity is missing in the standards, for which an uncertainty budget could be established following the procedures used in GUM (1995). For the test setup discussed here, the standard EN 61000-4-3 defines for example a virtual grid on which the electrical field strength distribution is measured without DUT as a function of frequency. When now the DUT is placed inside the field generator its coupling to the interior of the field generator is not determined at all. Considering the different structures of absorber-lined chambers (absorbing walls far away from the DUT) and GTEM cells (metallic reflectors close to the DUT) a different coupling between each of the FGs and the DUT is obvious. In Schrader (1997) it was shown that for a metallic box of $30 \mathrm{~cm}$ length without cabling the test severity varies by about $\pm 4 \mathrm{~dB}$, giving large influence on the measurement results. Obviously, the empty field strength is not a suitable parameter to compare the test severity. In a round-robin test among more than 100 accredited EMC test laboratories in Germany (Spitzer et al., 2003) using different types of FG a large spread of results was observed. It has been empirically shown there that the standards are not detailed enough in their given specifications to obtain reproducible and reliable test results, which can be assigned to the reasons described above.

But as one can immediately see, all standards aim at the same result - to generate a specified test severity. It is obvious, that the results must be comparable within their uncertainty margins, even though it might not be possible to convert the test specifications for the different test methods. These are often referred to as independent test methods. But 
in case they are not comparable at all, the question arises whether any of the methods represents the susceptibility of the DUT. The problem so far was to find a measurable quantity which subsumes all influences at once and could be linked to any independent reference. The deviation of measurements from the reference, which might be just a theoretical value, would help to determine the uncertainty for the actual test setup.

This work describes a method how to obtain a physical quantity which describes all influences on the measurement uncertainty for devices with metallic enclosures. Influences are, for example, the field strength level, the field structure (or 3-D-components), any near- or far-field effect, any coupling between the outer shell of the DUT to the interior of the FG, and any resonance inside the FG and / or inside the DUT. With this method it is rather simple to derive the uncertainty budget for EMC testing without burdening any systematic error of one particular (historic) method to any of the others. Some results obtained in different field generators are given.

\section{Theory}

In order to find any link between the electromagnetic field generated for a test purpose and the actual disturbance level, we look at the interfaces which can basically couple energy from the field to the electronics of the DUT possibly yielding a malfunction of the DUT. The second topic to be discussed is how to obtain the degree of equivalence of tests performed in different FGs. Combining both issues will guide us to a possible solution of our problem.

In order to explain our ideas, we are considering a metallic sphere in a free-space environment. Since many of the enclosures of DUTs are metallic or metalized cases with openings or cable entry points, this seems to be reasonable. The metal enclosure will at least serve as a worst-case situation and it reduces the problem level to a clear and manageable situation. We are assuming the skin depth being small compared to the thickness of the metalized layer of the sphere. That way we have only coupling through defined ports. Now, considering a plane wave impinging on the sphere being located in a free-space environment, surface currents and charges will be driven by the external electromagnetic field. Let us assume furthermore one simple slot with a high aspect ratio being the only path from the exterior to the interior of the sphere. We then can treat the slot as a "directional coupler", because it provides a high coupling factor into the sphere if the surface current has a direction perpendicular to the slot's largest dimension. For the direction parallel to the slot the coupling is much lower. Only the slot geometry determines the tensor which describes the coupling factor of the slot (Sturm, Römer, 2002). Therefore, the coupling factor will remain the same, even if the shielding effectiveness of an enclosure with one slot is measured in different FGs. However, if we consider the amount of energy coupled into the DUT, Schrader (1997) has shown in experiments, that the energy is also dependent on the different excitation, which is determined by the actual charge and current distribution on the surface of the DUT. Sturm and Römer (2002) described these empirically based data in a more formal way.

In case we are able to predict the coupling of a known source into the metal sphere (e.g. by numerical calculation), we only have to determine the excitation of the coupling port and the backward interaction from the sphere's interior to the exterior. It depends on the material's properties whether resonances will occur inside the DUT or not. This was one of the problems measuring the shielding effectiveness of RF protection suits. By filling those partially with absorbers or other lossy material the unwanted and misleading resonances (Klinkenbusch, 1998) are avoided. From now on we will use a closed sphere only, so we can disregard the backward interaction. This way we have separated the internal from the external problem. But still, the excitation of the problem is the surface current and charge distribution. If we determine those on a sphere with given diameter inside a particular FG, we are able to compare these results with those obtained analytically on a sphere of the same diameter in a free-space environment (Schrader, 1997 and 2002). Of course a suitable measurement system is needed, providing amplitude and phase information of a signal proportional to a surface current density distribution (Schrader, 1997).

To measure this, we have used a closed sphere with one surface current sensor mounted. The scattering of the sphere is invariant against rotation, so we can sample the current on the whole surface by just a simple rotation of the DUT. In our investigation we neglect an influence of the sensor itself on the currents and surface fields, respectively. This influence needs further investigation and will be subject of one of the next papers.

In order now to compare the currents, we segmented the surface into $N$ patch elements. For each patch we obtained the phasor for two orthogonal current components measured by the sensor and calculated by using the Methodof-Moments (Singer, Brüns, 2004). The amplitude and phase reference may be chosen arbitrarily on the DUT; we preferred the data measured on the patch being boresight to the transmitting antenna as reference.

The surface current sensor (SCS) is basically a half-loop antenna and evaluates the well-known equation

$\boldsymbol{J}=\boldsymbol{n} \times \boldsymbol{H}_{\text {tan }}$

with the surface current density $\boldsymbol{J}$, the normal vector $\boldsymbol{n}$ of the surface and the vector of the tangential magnetic field $\boldsymbol{H}_{\tan }$.

With this sensor we obtained the phasors of the measured orthogonal current components $\underline{J}_{\mu, i, f, F G},\left.\underline{J}_{v, i, f, F G}\right|_{N}$ for each patch $i$ on one particular sphere at the frequency $f$ in one particular FG (we have used a sphere with $50 \mathrm{~cm}$ diameter). Using "Concept" we calculated for each patch $i$ the orthogonal complex-valued phasors $\underline{J}_{\mu, i, f, F S},\left.\underline{J}_{v, i, f, F S}\right|_{N}$ for the free-space environment as the reference field generator. In order to determine the difference current vector for each patch $i$ we calculate first the phasor difference for both current components on each patch $i$

$\Delta_{\mu, i, f}=\underline{J}_{\mu, i, f, F G}-\underline{J}_{\mu, i, f, F S}$ 


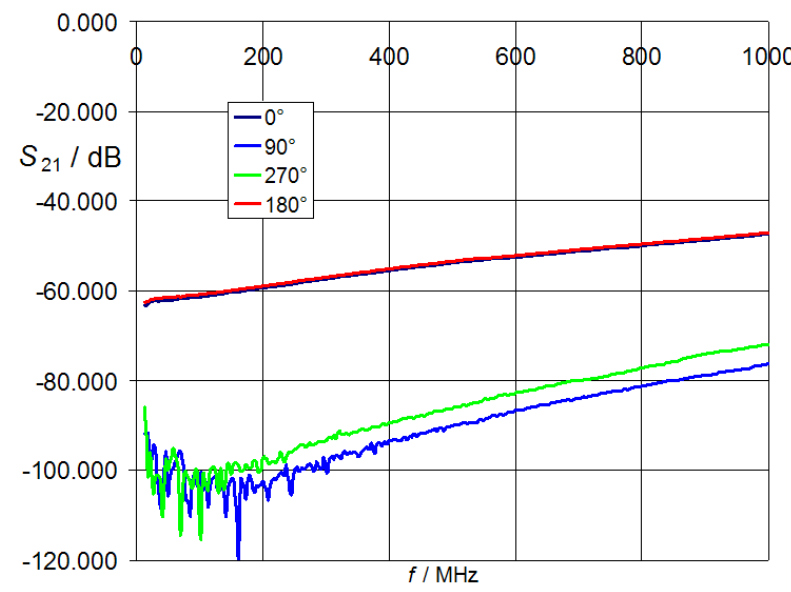

Fig. 1. Surface current sensor output in a $\mu$ TEM cell, where the cell's ceiling is replaced with the sensor's groundplane. For $90^{\circ}$ and $270^{\circ}$ the sensor is decoupled from the magnetic field, while for $0^{\circ}$ and $180^{\circ}$ the symmetry of the sensor can be accessed.

$\Delta \underline{J}_{v, i, f}=\underline{J}_{v, i, f, F G}-\underline{J}_{v, i, f, F S}$

The magnitude of the difference current vector for each patch $i$ can the be obtained by

$\left|\Delta \underline{J}_{i, f}\right|=\sqrt{\left(\Delta \underline{J}_{\mu, i, f}\right)^{2}+\left(\Delta \underline{J}_{v, i, f}\right)^{2}}$.

Using Eq. (4) we calculate now the mean deviation for all $M$ frequencies and for all $N$ patches $i$ according to

$\overline{\left|\Delta \underline{J}_{N, M}\right|}=\frac{1}{N} \cdot \frac{1}{M} \cdot \sum_{f=1}^{M} \sum_{i=1}^{N} \sqrt{\left(\Delta \underline{J}_{\mu, i, f}\right)^{2}+\left(\Delta \underline{J}_{\nu, i, f}\right)^{2}}$

It depends on the relationship between the size of the sphere and the frequency range how many patches and samples of the surface current distribution are needed to describe any deviation in one particular FG. This will be subject to further investigations.

It might be interesting to normalize each component of the current vector (Eqs. 2, 3) with the free-space values $\underline{J}_{\mu, i, f, F S}, \underline{J}_{\nu, i, f, F S}$, otherwise we have weighted the results by omitting contributions from areas of the surface being less excited from the direct electromagnetic wave.

We used the mean deviation according to Eq. (5) to evaluate which surface area is mostly effected by the FG. The relative spread of the deviation $r s d$ (Eq. 6) shows, whether a significant evidence is existent and if so, at which part of the surface it can be found.

$r s d(f)=\max _{i=1 \ldots N}\left(\frac{\left|\Delta \underline{J}_{i, f}\right|-\overline{\left|\Delta \underline{J}_{N, M}\right|}}{\mid \overline{\Delta^{\prime} \underline{J}_{N, M} \mid}}\right)$

The plot of $r s d(f)$ vs. frequency $f$ could give some insight, whether the influence is of narrow or broadband type. All of
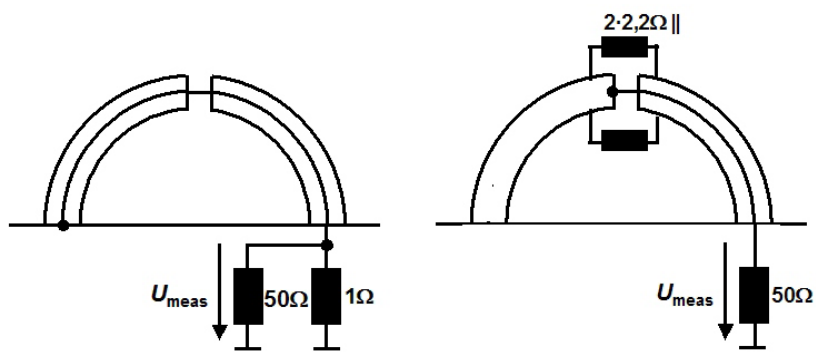

Fig. 2. Former (left) and new (right) design of the surface current density sensor.

these effects and correlations will be investigated in the future, but one can already be analyzed now, which is the ratio eff between the empty field strength $\underline{E}_{x, y, z, f}$ measured with the E-field sensor at locus $x, y, z$ in the FG and the surface current $\underline{J}_{\zeta, \vartheta, \varphi, f}$ on the patch $i$ of the sphere in the same FG.

$\underline{e f f}=\frac{\underline{J}_{\varsigma, \vartheta, \varphi, f}}{\underline{E}_{x, y, z, f}}$

The result of Eq. (7) can quasi be treated as an efficiency, namely to which extent the electrical field strength (which is the only indication for test severity in EMC testing now) is converted to a surface current on a DUT (which is the more important physical value to obtain uncertainty and comparability).

\section{Measurement Setup}

To obtain surface current distributions on a DUT a probe system based on a fiber-optical link was used here (Schrader, 1997). It is implemented in a loop (measurement setup) consisting of a vector network analyzer (VNA), a power amplifier and a transmitting antenna in a semi-anechoic chamber as FG. The scattering parameter of forward transmission $S_{21}$ is measured and stored in the frequency range from $80 \mathrm{MHz}$ to $1000 \mathrm{MHz}$.

To avoid any influence of power variations the actual forward power can be taken into account using a 4-samplerVNA. Instead of the reflected incident signal $\underline{b}_{1}$, we feed the forward power branch of a directional coupler as input quantity into the appropriate channel of the VNA.

The surface current sensor (SCS) consists of a half-loop antenna over its "groundplane". Usually a thin semi-rigid cable is used as half-loop, with the outer conductor cut along the circumference. As the cut is symmetrical, the $E$-field influence is reduced by balancing the sensor signal. To show this, results were obtained in a $\mu$ TEM cell, where its ceiling was replaced by a sensor's groundplane. By rotation of the groundplane of $90^{\circ}, 180^{\circ}$ and $270^{\circ}$ the change in the sensor output is observed. As it can be seen in Fig. 1 the suppression of $E$ is better than $20 \mathrm{~dB}$ up to $1 \mathrm{GHz}$. The sensor was not calibrated during our investigations, but we recommend a calibration traceable to the SI units using a $\mu$ TEM 


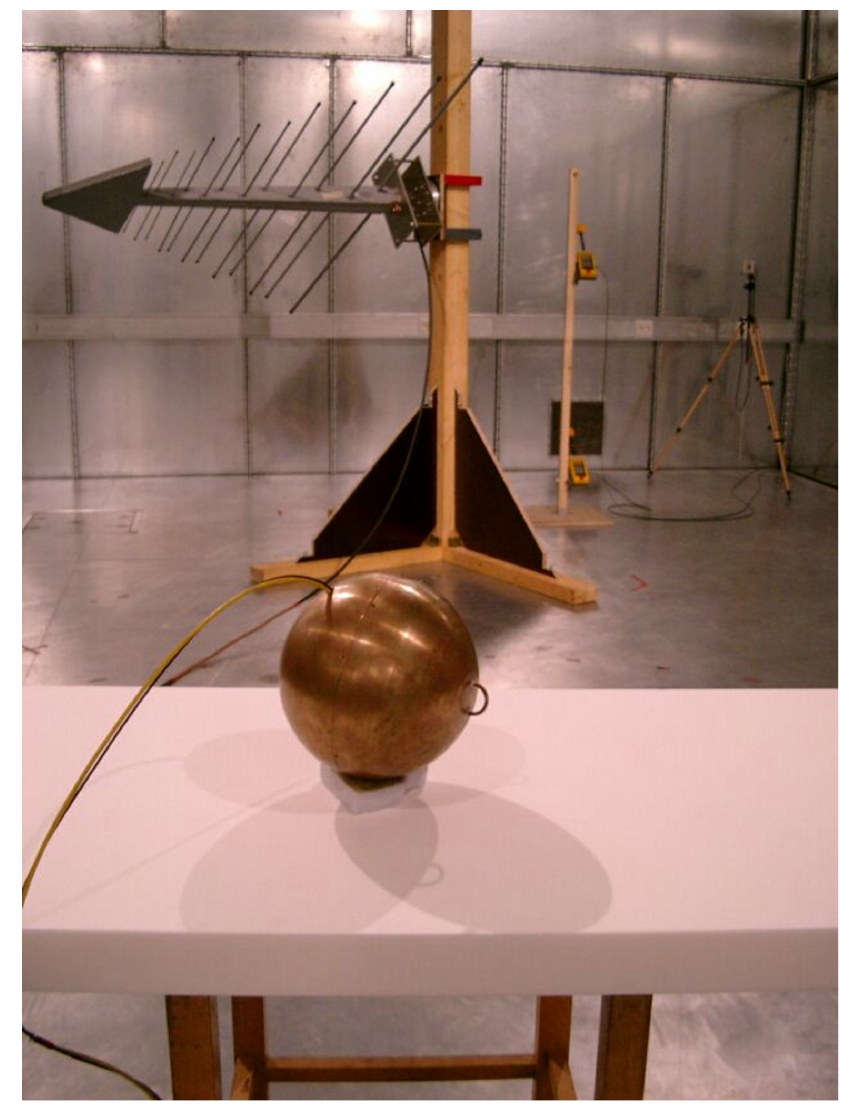

Fig. 3. Test setup in a Reverberation Chamber (TU Braunschweig). A logarithmic-periodic antenna (LPA) serves as transmitter, the surface current sensor is mounted on a sphere with $18 \mathrm{~cm}$ diameter (PTB Braunschweig). The metal sphere contains the electronics, only the fiber-optical link is penetrating the surface. The half-loop antenna can be seen in the front of the sphere at the right side. The transmitting LPA is not boresight at all.

cell. A noticeable improvement of the sensor's response was achieved by a new design. We have avoided the transforming parts of the shortened branch of the coaxial cable (see left side of Fig. 2). In the new design (right side of Fig. 2), the measurement signal across the resistor of $1.1 \Omega$ is fed into the $50 \Omega$ load of the network analyzer by a $50 \Omega$ cable. Thus, the frequency response is smoothed and we obtain a constant response up to at least $3 \mathrm{GHz}$.

It was interesting to compare the results according to Eq. (7) obtained inside a reverberation chamber (FVK) with results obtained inside a semi-anechoic chamber (SAC) with additional absorbers on the ground. As the field structure in a FVK should be statistical, $E$ and $J$ can be measured at an arbitrary orientation of both sensors and at an arbitrary location $[\mathrm{x}, \mathrm{y}, \mathrm{z}] \neq[\varsigma, \vartheta, \varphi]$. The transmitting antenna will not be boresight at all (Fig. 3). The FVK tuner is set in 50 different orientations (steps of $7,2^{\circ}$ ) in a $360^{\circ}$ rotation. For calculating eff according to Eq. (7) in this case the maximum value out of 50 samples for $E$ and $J$ was taken, respectively. For the setup in the SAC the transmitting antenna and the E-field probe were oriented in the same polarization. The coupling

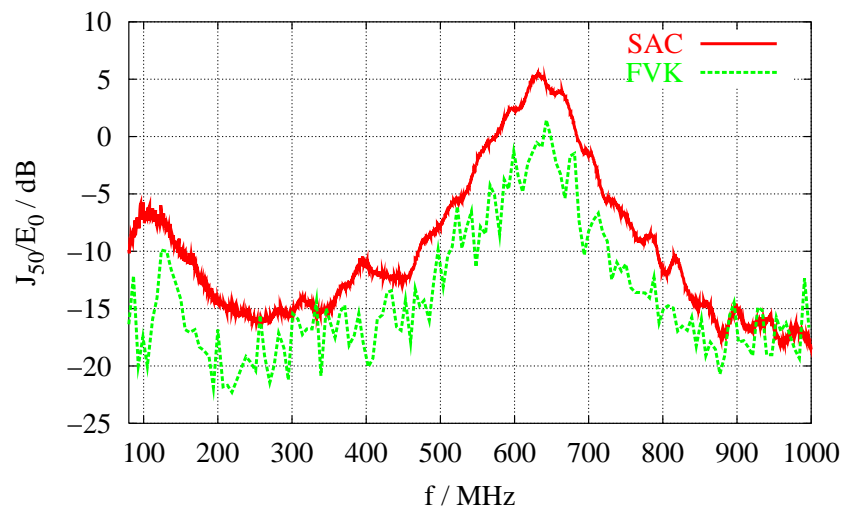

Fig. 4. Measurement results of the ratio $J / E$ with the surface current density $J$ and the empty field strength $E$ obtained inside a reverberation chamber (FVK) and inside a semi-anechoic chamber (SAC) with additional absorbers on the ground. The calculated mean deviation between both curves is about $6 \mathrm{~dB}$.

to the surface current sensor mounted on a sphere with $18 \mathrm{~cm}$ diameter was maximized by setting the normal vector of the plane of the half-loop receiving antenna being parallel to the magnetic field. The result calculated according to Eq. (7) is shown in Fig. 4. For the FVK we have found eff to be about $6 \mathrm{~dB}$ less than for the SAC. It can be explained by the degrees of freedom for the energy or, from a practical point of view, by the fact that the E-fields in the FVK are measured in a standing-wave environment (Eulig, 2004). Placing the metallic DUT inside the FVK will move the boundary condition, but does not change the standing wave situation (comparable to a stirr). Inside the empty SAC with additional absorbers on the ground we have a field structure close to a far-field situation (plane wave in free-space environment). Placing the DUT inside the SAC we change the field into a standing-wave setup, which gives a factor of 2 for the tangential magnetic field strength $H_{t a n}$. For practical applications this means that the field strength in a FVK has to be chosen $6 \mathrm{~dB}$ higher than in plane-wave-FGs in order to gerenate comparable testing conditions. We will investigate this result further.

\section{Conclusion}

EMC tests are carried out in different field generators, for which different standards are available. When all setups are aiming at the same goal, results must be comparable within their uncertainty margins. To be able to obtain the measurement uncertainty of radiated EMC tests, a suitable physical quantity has to be chosen, for which an uncertainty budget can be established following the well-known procedures from the GUM (1995). We have shown, that surface current densities on a simple object like a sphere in a free-space environment can be treated analytically as a reference, while surface currents can be measured on such an object in a real field generator. A comparison of both results allows to determine any deviation between reference and actual field generator 
without burdening any systematic error of one particular (historic) method to any of the others. Comparing the ratios of $J / E$ obtained in the SAC and in the FVK, a deviation of $6 \mathrm{~dB}$ is observed, which can be explained by the degrees of freedom of the energy.

\section{References}

Eulig, N.: Eignung der Feldvariablen Kammer (FVK) für EMVStörfestigkeitstests, Dissertation TU Braunschweig, Shaker Verlag, Aachen, ISBN 3-8322-2945-0, 2004.

Guide to the Expression of Uncertainty in Measurement (GUM), first edition 1993, corrected and reprinted 1995.

IEC EN 61000-4-3 (EMC) Part 4-3: Testing and Measurement Techniques - Radiated, radio-frequency, electromagnetic field immunity test, Basic EMC publication, 2002.

IEC EN 61000-4-20 (EMC) Part 4-20: Testing and Measurement Techniques -, Emission and Immunity testing in transverse electromagnetic (TEM) waveguides, 2000.

IEC EN 61000-4-21 (EMC) Part 4-21: Testing and Measurement Techniques -, Emission and Immunity testing in Reverberation Chambers, 2003.

IEC EN 61000-6-1 (EMC) Part 6-1: Generic Standards - Immunity for Residential, commercial and light-industrial environments, 2005.

IEC EN 61000-6-2 (EMC) Part 6-2: Generic Standards - Immunity for industrial environments, 2002.

IEC EN 61000-6-3 (EMC) Part 6-3: Generic Standards - Emission testing for Residential, commercial and light-industrial environments, 2002.

IEC EN 61000-6-4 (EMC) Part 6-4: Generic Standards - Emission testing for industrial environments, 20021.

International Organization for Standardization (ISO).

Klinkenbusch, L.: Multipolanalyse des Schirmverhaltens bei transienter Störaussendung, in: Elektromagnetische Verträglichkeit EMV '98, edited by: Schwab, A., Berlin-Offenbach: VDEVerlag, 169-176, 1998.

Schrader, T.: Vergleich von Feldgeneratoren für EMV-Prüfungen, Dissertation TU Braunschweig, Shaker Verlag, Aachen, ISBN 3-8265-2782-8, 1997.

Schrader, T.: A Semi-Analytical Method for the Determination of the Severity of Tests in Measurements of the Immunity to Electromagnetic Interferences (EMC), Technisches Messen, 69, 2, 70-76, 2002.

Singer, H. and Brüns, H.: Concept II: Code for the Numerical Computation of Electromagnetic Processes for Thin Wire and Thin Shell Structures Including Dielectrics, Department of Theoretical Electrical Engineering, TU Hamburg-Harburg, 2004.

Spitzer, M., Münter, K., Pape, R., Glimm, J., and Dallwitz, L.: EMC-intercomparison by DATech/RegTP/PTB - results, findings and conclusions, Technisches Messen, 70, 3, 151-162, 2003.

Sturm, R. and Römer, B.: Measuring the Leakage of Gaskets, Technisches Messen, 69, 2, 102-107, 2002. 